\title{
Identidad y lengua en el ciberespacio: ¿existe una conciencia lingüística digital?
}

Juan-Manuel López-Muñoz

\begin{abstract}
Resumen
En el orden del ciberespacio, muchos internautas se denominan a símismos nativos o indígenas digitales, constituyendo una nueva comunidad étnica ajena a las fronteras territoriales y culturales del mundo físico, unidos por lo que podríamos llamar una misma "conciencia digital". En este artículo estudiamos cómo se manifiesta esa conciencia digital a través de las expresiones que los usuarios de Twitter emplean para hablar de quiénes son y qué hacen cuando comunican por ordenador o por otros dispositivos conectados a la red de Internet. Nuestro objetivo es intentar sistematizar algunas características específicas de la lengua digital, con el fin de proponer algunas pistas para la pedagogía de la lengua digital y participar en el debate de "buen uso" de la lengua digital en el marco de la cortesía digital.
\end{abstract}

Palabras-clave: lengua digital; conciencia digital; cortesía digital; Twitter.

Recebido em: 15/01/2019 Aceito em: 11/03/2019

\footnotetext{
a Professor do Departamento de Francês da Universidade de Cádiz (Espanha). E-mail: jmanuel.lopez@uca.es.
} 
Comme le français, je parle numérique tous les jours.

Les écrans sont mes nouvelles maisons.

Je les adore mais loin de m'y poser, ils me dévorent...

[Chrystel Egal, http://c-egal.com]

1 Prensky (2001) distingue los "Digital Natives", nacidos a partir de los años 90 , en la llamada era digital, de los "Digital $\mathrm{I} \mathrm{m} \mathrm{m}$ igra $\mathrm{nt} \mathrm{s}$ " personas nacidas antes de ese periodo y que están más o menos familiarizadas con las nuevas tecnologías de la comunicación.

2 "je ne vais plus sur internet, j'y suis dedans jusqu'au cou" cf. https:// c-egal.com/ecrana-cran-ou-adorationdevoration/
Haya o no consenso de carácter científico a la hora de admitir, hoy en día, la existencia de nativos digitales, desde que M. Prensky ${ }^{1}$ acuñara este término en 2001, y por tanto de admitir la existencia de una lengua digital, lo cierto es que hablamos digital todos los días. La lengua digital se va imponiendo poco a poco como lengua dominante (HAGĖGE 2011, p.21), mano a mano con el inglés comercial enriquecido con elementos plurisemióticos, con nuevas normas y nuevas prácticas.

Al margen de lo que digamos los científicos, en el orden del ciberespacio, muchos internautas se denominan a sí mismos nativos o indígenas digitales, constituyendo una nueva comunidad étnica más o menos ajena a las fronteras territoriales y culturales del mundo físico, caracterizada por lo que podríamos llamar una conciencia digital. Basta navegar un poco por Internet para encontrar numerosos ejemplos de perfiles de individuos y asociaciones que dicen explícitamente su pertenencia a tal tribu.

En la cita que sirve de epígrafe a este artículo, extraída de un poema de la artista multidisciplinar Chrystel Egal, la poeta se declara bilingüe francés y digital al afirmar que "habl[a] digital todos los días, igual que el francés". Las pantallas son un territorio ("sus nuevas casas") no sólo por el que transitar, sino donde vivir y experimentar con los cinco sentidos ${ }^{2}$. Nótese que la artista, al destacar la frecuencia cotidiana con la que habla francés y digital, a la vez compara y distingue ambas lenguas, mostrando además una marcada relación emocional con ésta última.

Así, podemos afirmar que existe, al menos, una conciencia de la diferencia entre la lengua que usamos cuando estamos conectados a Internet y la que aprendimos de nuestros padres y en la escuela. Esta conciencia se manifiesta a través de las expresiones que usan los internautas para describir la lengua que usan en el ciberespacio y para hablar de quiénes 
son y qué hacen cuando (se) comunican por ordenador o por otros dispositivos interconectados. Las posturas parecen oscilar entre aquellos que consideran lo digital como un rasgo esencial y los que lo tratan como una competencia adquirida. $Y$ de esta manera, podemos encontrar ejemplos, como el de este usuario de Twitter que se muestra aquí abajo, en cuya biografía la relación con lo digital se expresa en términos de una necesidad vital, cotidiana, como alimentarse y beber agua. Desde esta perspectiva, ser nativo digital no solo es saber usar las tecnologías de la información y la comunicación y, sobre todo, saber hablar de ellas, sino pasar la mayor parte del tiempo conectado a Internet, hasta el punto de identificarse con la máquina. "La mayor parte del tiempo soy un [ro]bot", afirma este @NativoDigital:

Fig. 1

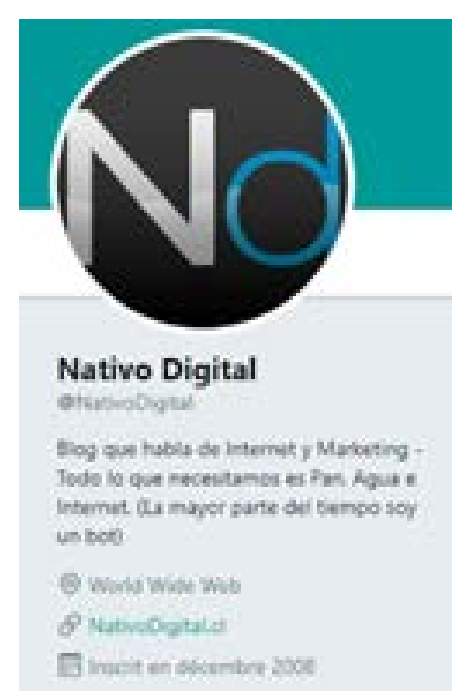

En estos otros ejemplos, también sacados de Twitter, ser nativo digital se entiende, en cambio, como una competencia profesional añadida, en cierto modo sinónimo de Techie (apasionado por la tecnología) y de Tech-savvy (conocedor de la tecnología moderna, especialmente de los ordenadores): 
Fig. 2
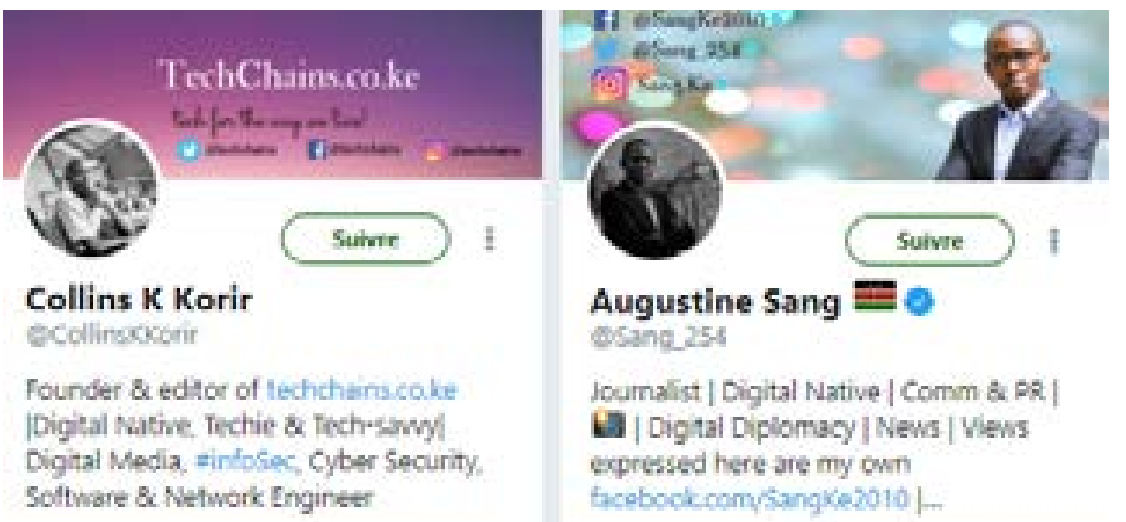

Augustine Sang $\equiv$ esane.25s

Joumalist | Digital Native | Comm \& DR |

B] Digtal Diplomacy | News | Views expressed here are my own tacebock comysangked010 1...

El ejemplo siguiente es particularmente interesante porque apunta no sólo al carácter innato de las competencias digitales en "todos aquellos nacidos con la tecnología", sino a un potencial de producción lingüística, expresado metafóricamente con el término de "fuente". Obsérvese el empleo de la preposición "con" en la expresión "nacidos con la tecnología" que pone de relieve el aspecto relacional más que simplemente espacial del entorno tecnológico. Volveré sobre esto más abajo. Por otra parte, el uso del pronombre "todos" y del nombre de usuario en plural, @Nativosdigitais, destaca el rasgo colectivo de la identidad digital.

Fig. 3
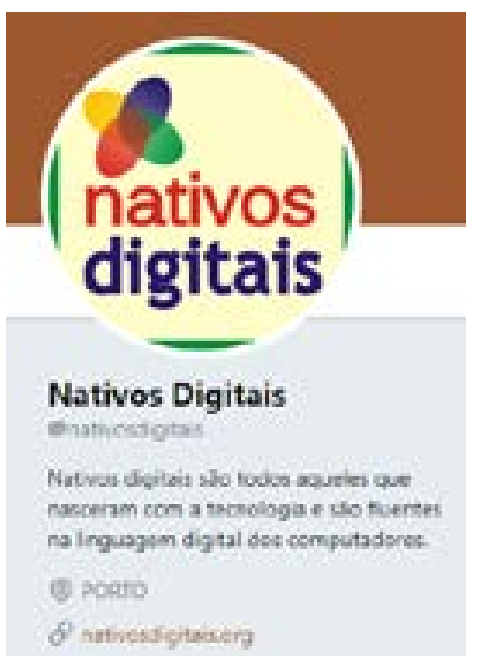
El carácter fundamentalmente colectivo de la identidad digital afecta incluso al propio concepto de individuo, cuya unidad se diluye y multiplica en diversos avatares discursivos en el ciberespacio. Esta usuaria de Twitter cuyo perfil se muestra a continuación ha elegido, como imagen de cabecera de su cuenta, la foto del reflejo en el espejo de un personaje de ficción cinematográfica ${ }^{3}$, Quorra, una joven ISO (algoritmo isomórfico) de aspecto andrógino, perteneciente a una raza generada espontáneamente en y por el sofisticado universo cibernético del videojuego de Tron. La imagen redunda en la idea expresada en la biografía del perfil, en la que la usuaria se hace llamar@numérique ${ }^{4}$ y se describe como siendo "cientos de seres":

Fig. 4

${ }^{3}$ Se trata del filme estadounidense "Tron: Legacy" de 2010, dirigido por Joseph Kosinski a partir de un guión de Adam Horowitz y Edward Kitsis, y distribuida por Walt Disney Pictures.

${ }^{4}$ La lengua francesa usa preferentemente el término "numérique", como adjetivo y sustantivo (procedente del latín numerus), en los contextos en los que el resto de las lenguas europeas emplean "digital" (del latín digitus). Algunos estudios señalan (MOATTI, 2016), no obstante, la tendencia creciente en la lengua francesa a adoptar este último término, así como su derivado "digitalisation", en lugar de "numérique" y "numérisation".

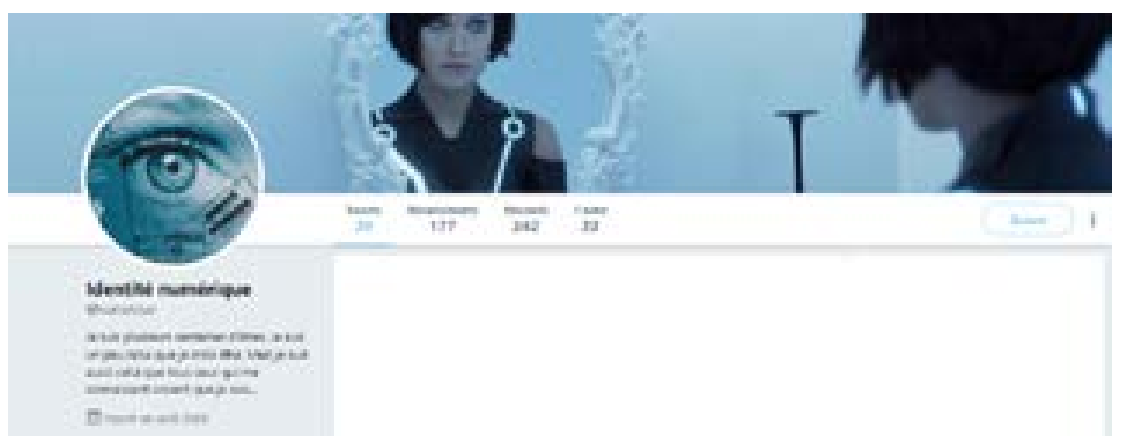

Según se desprende de los discursos de los internautas, la propiedad de lo digital se extiende, más allá de la identidad de los hablantes digitales, a todas las facetas de lo humano. En la siguiente selección de perfiles, vemos el epíteto digital caracterizando a la sociedad, la ciudad, la cultura... e incluso un café: 
Fig. 5

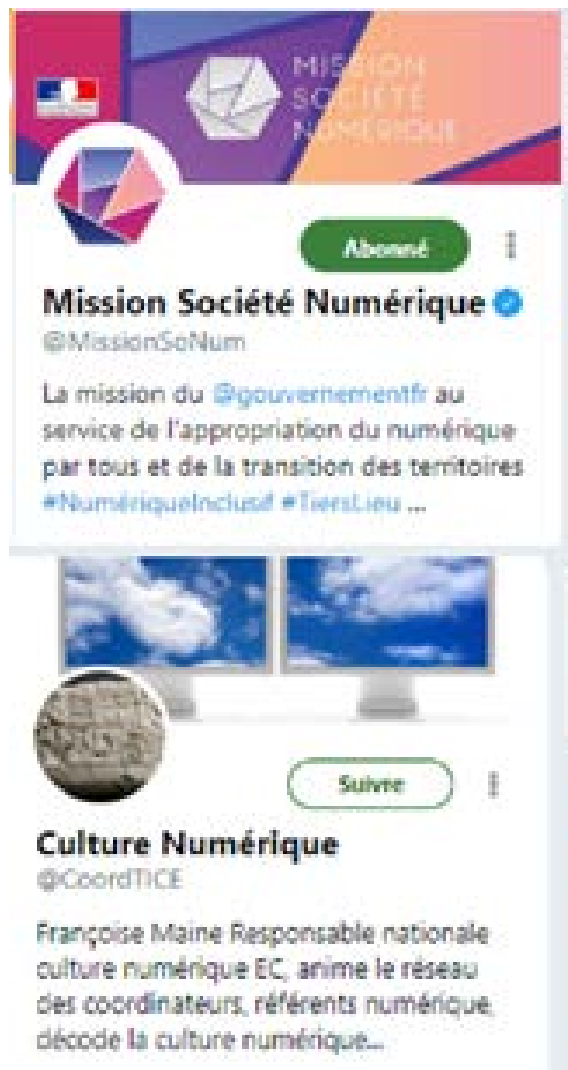

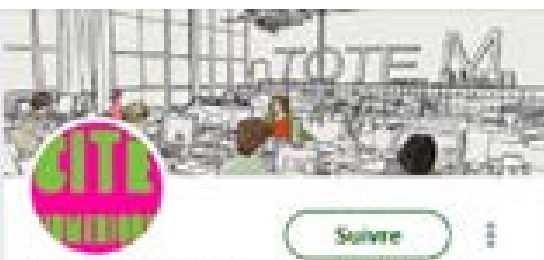

Cité Numérique

eccitetumerique

Compte ctficiel de ta cite Numerique, bátiment-totem de la efienchifechiox: ouverture debut 2018

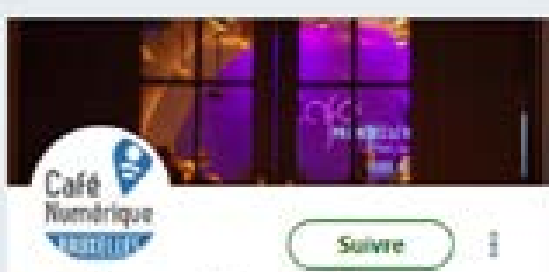

Café Numérique BxI ocateverd

Curieux et passionnes par les nouvelles technologies et Rinnovation ? Rejoignez. nous egalement sur Fasebook facebock coms calenbruxelles

Lo digital parece abarcar, según esto, toda aquella actividad humana que, en un proceso adaptativo al entorno de las tecnologías de la información y la comunicación, se manifiesta por un comportamiento, unos objetivos y unos usos diferentes respecto a los que empleamos en situaciones de interacción en el mundo fuera del ciberespacio.

La interacción con la máquina es compleja, pues la máquina no es solo un objeto mediador que posibilita la búsqueda de información y la comunicación a distancia entre individuos, sino que es también fuente de un nuevo real (la realidad virtual, el ciberespacio), además de poder funcionar como interlocutor, colaborando en la realización de tareas solicitadas por el hablante digital. Comunicar por ordenador implica "utilizar" la máquina, más que simplemente usarla (TRICOT et al., 2003), es decir, implica construir un conjunto de interacciones a modo de interfaz entre los sujetos, la máquina y la sociedad. 
La diferencia entre usar la máquina (en sentido de servirse de la máquina) y utilizarla es que esta acción conlleva consigo una idea de utilizabilidad, y por tanto una potencialidad de apropiación (LATZKO-TOTH \& PROULX, 2015, p.24) además de una idea de accesiblidad, (teniendo en cuenta factores como coste, compatibilidad, fiabilidad) $\mathrm{y}$, en fin, de aceptabilidad respecto a los comportamientos y los roles sociales establecidos (BÉTRANCOURT 2007, p.77).

Y aunque se trata de utilizar máquinas, investigadores como Woods \& Decker (2000) subrayan que el manejo no es mecánico, sino que requiere un proceso adaptativo constante en ambos sentidos: del humano respecto a la máquina y de la máquina (el diseño y las propiedades de esta) respecto a las necesidades e intenciones de los humanos. En cuanto al primer caso, esta adaptación del humano a la máquina se manifiesta, por citar un ejemplo simple que cualquiera de nosotros ha podido experimentar, en la manera en que ajustamos el léxico, la sintaxis, la entonación o el ritmo en tareas de búsqueda de información en Internet, mediante voz o teclado, para garantizar la consecución de nuestros objetivos, en función de los recursos que ofrece la máquina.

En tal proceso de ajuste, al igual que ocurre en las interacciones comunes fuera del ciberespacio, las competencias y, por tanto, la probabilidad de éxito de la interacción, varían en función de las categorías socioculturales y profesionales de los hablantes. En efecto, algunos autores puntualizan que la familiaridad actual con las máquinas no es sinónimo de apropiación real de las competencias digitales (BROTCORNE et al., 2010, BOURDELOIE 2012, FOUCHER y YUN 2016). Y así, podemos distinguir con Baron y Bruillard $(2008$, p. 9) a los "expertos digitales innatos" de los "nativos ingenuos" $\mathrm{o}$ usuarios que usan de la tecnología frecuentemente, pero en un espectro muy limitado y con un grado de autonomía relativa debido a su escasa cultura digital pese a haber nacido y crecido en un entorno tecnologizado.

En consecuencia, parece que la identidad digital implica empleado por los autores citados es "digital naïves"; se trata de un juego de palabras a partir del término común de "digital natives".

no solo vivir e interactuar en un entorno tecnologizado sino también poseer una cultura digital y una capacidad o disposición para apropiarse la lengua digital. 
Respecto a ello, Foucher y Yun $(2016$, p. 3) sostienen que es preciso distinguir las competencias digitales naturales de las adquiridas en un contexto educativo, y sitúan las competencias digitales en una escala de gradación que va de la simple utilización o el simple consumo de las máquinas a la apropiación de las competencias digitales a nivel de experto digital, en la que intervienen factores principalmente sociales y económicos. Estas mismas investigadoras apuntan a la existencia de un hiato entre las prácticas privadas, por un lado, y las escolares, académicas o profesionales, por otro, siguiendo una lógica que, citando a Guichon (2012), califican de apropiación en el primer caso, y de imposición en el segundo.

¿Podemos deducir de lo anterior la existencia de dos registros de lengua digital diferentes, uno más o menos culto o institucional y otro más o menos coloquial o familiar? Autores como Peraya y Bonfils (2014, p. 247) y Klein (2012, p. 112) sostienen que estas dos esferas de utilización no deben entenderse como compartimentos estancos, sino que están separadas por fronteras permeables que permiten la circulación de prácticas en ambos sentidos, en determinadas condiciones tecnológicas y socioculturales, en función de las representaciones que los hablantes digitales se hacen de la lengua digital y de su(s) identidad(es) digital(es).

En realidad, lo que parece estar en juego aquí no es la cuestión de la aceptación de la existencia de la lengua digital, la cual que podría caracterizar a los nativos digitales, sino la cuestión de la pertinencia de los criterios que empleamos a la hora de comprender la relación entre lengua y habla, entre corrección lingüística y felicidad pragmática del acto de comunicación. Pues, ¿acaso todos los nativos de cualquier lengua, por el simple hecho de ser nativos en esa lengua, conocen y saben aprovechar las potencialidades de su lengua al mismo nivel? Así, considerarse nativo digital parece implicar más un sentimiento de pertenencia a un grupo, en el plano social y afectivo, que la posesión de un nivel de maestría en la lengua digital o de experto en el aprovechamiento de las herramientas digitales, los cuales se adquirirían a través del estudio, como en el caso de cualquier otra lengua.

Visto desde una perspectiva lingüística tradicional, lo digital suele considerarse un habla más que una lengua, es 
decir, un modo (contemporáneo y dominante) de comunicación más que un código lingüístico particular. Es bien sabido, no obstante, que el habla digital parte de un código propio, el código binario informático, de carácter numérico (de ahí la expresión "digital", derivada de dígito), concebido para el tratamiento racional de la información, es decir, con una función meramente informática. Ahora bien, poco a poco, con la generalización de Internet y a medida que ha evolucionado el concepto mismo de información, este código ha demostrado su capacidad no solo para "traducir" a números los idiomas existentes, sino al mismo tiempo para transformar las prácticas comunicativas y construir una nueva red de relaciones entre códigos semióticos, nuevas significaciones sociales, nuevas metáforas y nuevos mitos (Musso, 2008), hasta el punto, en fin, de dar lugar a lo que hoy conocemos como una cultura "característica de Internet", con su propio patrimonio, y sus "posibilidades inéditas de experimentación de la identidad", tal como reconoce la UNESCO en su informe mundial Hacia una cultura del conocimiento de 2005 .

Así, la lengua digital aparece a la vez como un sistema de relaciones entre códigos semióticos y como un fenómeno social que no dependen de (no están subordinados a) una determinada nacionalidad ni a una determinada cultura pretecnológica, sino que, atravesando las distintas lenguas y culturas previamente existentes, son capaces de construir su propio patrimonio, el patrimonio cibercultural, cuyos archivos (en el sentido foucaultiano) sustituyen lo escrito y lo oral por los nuevos registros del con(pu)tar con los dedos mediante los diversos objetos como el ordenador, el teléfono o la tableta. Esta cibercultura ya no es una mera masa de información, sino una amalgama de conocimientos adquiridos, de tecnoimaginario (BALANDIER, 1985) y de una memoria que se fabrica día a día con los discursos producidos y encontrados en la red, a la que se añade un saber hacer informático, mediático, personal, relacional y profesional. La cibercultura configura nuestro estilo de vida contemporáneo, nuestras organizaciones personales, familiares o profesionales, dentro y fuera de la red, en un gran proceso de "conversión digital" (DOUEIHI, 2008),

${ }^{6}$ Véanse las págs. $57 \mathrm{y}$ 58 para la versión en español. determinando nuestra relación con nosotros mismos, con el tiempo, con el territorio y con la sociedad. 
Lejos de servir simplemente para el almacenamiento e intercambio de datos, la lengua digital tiene ante todo una función social, cultural e incluso civilizacional (PLANTARD 2014, p. 44-47), sirviendo a la construcción de una nueva humanidad digital $y$, por ende, de un nuevo humanismo digital (DOUEIHI, 2011).

Por otra parte, cabe también esperar en este concepto de cibercultura la existencia de una multiculturalidad, ligada a una diversidad de comunidades o tribus virtuales (RHEINGOLD 1995; HARVEY, 1995; LEVY, 1997; MARCOTTE, 2003): piratas, ciberpunks, anonymous, geeks, nerds, etc., cada una con sus propios sistemas de signos y de relaciones, con sus propios ritos de interacción, de admisión y de expulsión, con sus propios mitos, así como cabe esperar la posibilidad de relaciones interculturales entre distintas culturas digitales y de estas con otras culturas anteriores y contemporáneas.

Por otra parte, admitamos o no la existencia de una lengua digital como tal, propia de los nativos digitales, no hay duda de que los usos en la comunicación mediada por ordenador han puesto en jaque no sólo los límites entre las lenguas, sino también entre diferentes códigos semióticos. Y sobre todo obligan a replantearse el concepto de lengua. ¿Qué características específicas debería tener la lengua digital para ser considerada legítimamente como una lengua? ¿Bastaría definirla como una práctica cultural diferenciada, o efectivamente constituye un sistema con sus propias normas distintas de las de otras lenguas? La respuesta a tales preguntas pasaría por un replanteamiento también de lo que entendemos por conciencia lingüística. ¿La conciencia digital es una propiedad innata o podemos cultivarla igual que cultivamos la conciencia cívica o la ecológica?

Es indiscutible que la lengua digital parte de códigos ya existentes, que le sirven de modelo y de garante, con los que construye usos diferenciados (ortográficos, sintácticos, textuales) sobre la base de la creación espontánea individual de los internautas y de las innovaciones tecnológicas. Y sabemos que, como en la interacción común fuera del ciberespacio, los gestos también son importantes, no solo las palabras, pero que estos ya no son semióticos por sí mismos, sino en tanto que dejan "trazas" en el periférico de entrada (CAELEN 1996: 16), 
7 Baste, a modo de ejemplo de síntesis de las formas y prácticas discursivas digitales, el diccionario publicado por Marie-Anne Paveau bajo el título de L'Analyse du discours numérique (HERMANN, 2017).

${ }^{8} \mathrm{Si}$ bien hay creadores de normas digitales, dictadas por actores socioeconómicos con gran poder de influencia en los medios sociales (PATEL, LOPEZ, PARTALAS \& SEGOND, 2017), no parece haber, por el momento, defensores de esas normas, al menos en el ámbito académico. Se observa, eso sí, una influencia creciente de lo digital en los manuales de gramática de las lenguas (BOYER, LEBRUN \& ROY, 2017). mediante la activación de botones en el teclado o en el ratón y de iconos en la pantalla.

Es igualmente evidente que la relación entre humanos y máquinas ha hecho posible una nueva forma de estructurar y organizar la comunicación, con nuevas potencialidades, nuevas formas de escribir e interactuar en línea, como no cesan de demostrar los numerosos trabajos que se publican cada día en el ámbito del Análisis del discurso digital ${ }^{7}$.

Y así, en tal estado de cosas, la conciencia lingüística digital no parece tener ya nada que ver con un sistema lingüístico convencional, institucionalizado, abstracto, de formas normalizadas, sino con la totalidad de las enunciaciones posibles en el entorno del ciberespacio. Tal concepción, en realidad, no es nueva: ya decía Bakhtine que la conciencia lingüística no se preocupa para nada de la forma de la lengua como tal, ni siquiera de la lengua misma (1929/1977, p. 102). El código y su sistema no es lo que definen a una lengua, sino que permiten, en todo caso, describirla y enseñarla. La conciencia digital existe desde que admitimos la existencia de la (inter-)acción digital, y se consolida cada vez que el hablante digital mira retrospectivamente a su producción digital, comprendiendo su participación en una Historia en train de se faire, con un horizonte de actuación (WALLON, 2009).

La conciencia lingüística digital se manifiesta, en fin, también a través del rechazo expresado implícitamente en las metáforas y representaciones líquidas con las que las culturas tradicionales describen la comunicación digital (BERNARDOT, 2018), demostrando con ellas el miedo a perder su poder simbólico terrestre.

Si la lengua digital parece carecer de su propia gramática ${ }^{8}$, podemos afirmar sin riesgo de equivocarnos que posee su propia lógica de uso (PERRIAULT, 1989), su propio sistema de interpretación (STIEGLER, 2014; FALGAS, 2018) y, en fin, sus propias textualidades (MARCUSCHI, 2001a, 2001b).

El texto digital no es lineal ni tiene unidad en sentido estricto, tradicional, sino que figura en el centro de una red de textos. Los enunciados digitales se caracterizan por su cibertopografía, su accesibilidad ilimitada, su plurisemiosis, su interactividad, siguiendo un nuevo paradigma de difusión textual más que de producción textual. O mejor dicho: un nuevo 
paradigma que rompe las fronteras entre la producción y la difusión en tanto que estos dos procesos están inscritos en una dinámica circular: se producen textos expresamente para viajar vinculados a otros textos, de manera que las fronteras entre proceso y producto, entre soporte y textualidad, entre prácticas lectoras y prácticas escritas, se confunden (como sostenía MARCUSCHI, 2001a, 2001b) o, mejor, se han vuelto porosas.

Caracterizada por la braquilogía, la fragmentación, la reutilización y la retextualización, la lengua digital se construye día a día sobre una red de escritura y lectura que está en constante construcción, a través de hipervínculos heterogéneos (imágenes, sonidos, palabras), y en constante renegociación, atendiendo a factores lógicos, afectivos y de proximidad. Por la red se navega, se ojea, se explora, se lee de un modo diferente (ASSOULINE, 2012), incluso con una postura corporal diferente (SZUNDY \& NASCIMENTO, 2016, p. 356), pasando de un texto a otro, unas veces con un proyecto de navegación, es decir, con un destino concreto a través de rutas más o menos guiadas, o bien sin proyecto definido, en un proceso de broutage o butinage (RHÉAUME, 2001, p. 194).

En cualquier caso, y pese a sus prácticas más o menos diferenciadas, la lengua digital comparte con las demás lenguas, además de sus códigos normalizados, el hecho de otorgar al hablante un espacio y un tiempo, y comporta, como ellas, un poder, un saber, una memoria, lazos sociales y prosperidad económica (SCARDIGLI, 1992, p. 50 et seq.), de ahí la importancia de la enseñanza y el aprendizaje digital.

Sabemos que la enseñanza/aprendizaje ${ }^{9}$ de lo digital está ligada a políticas públicas de educación que, a su vez, están determinadas por las representaciones de la tecnología en la sociedad. Hasta el momento, esta educación se ha centrado principalmente en el aspecto utilitarista de la máquina, como recurso o complemento para la docencia, con objetivos profesionalizantes, pero no propiamente en su dimensión identitaria, social y cultural. Parte de este problema, según señala Buckingham (2010, p. 40-41), está en la forma en que se

${ }^{9}$ Para una aproximación histórica y crítica de la enseñanza digital, véanse, entre otros, Buckingham, 2003; Duguet, 2014 y Siemens et al., 2015. ha asignado la inversión: la mayor parte de las financiaciones ha sido en infraestructura y en hardware, significativamente menos en la formación de profesores, persiguiendo unos objetivos a la vez consumistas y de conocimiento más que unos propósitos sociales. 
Personalmente pienso que la educación digital debería, en adelante, estar destinada no tanto a enseñar a "usar" la máquina como a mejorar la utilización de esta, es decir, a enseñar la lengua digital y los comportamientos lingüísticos digitales con vistas a mejorar las competencias pragmáticas de los internautas. Esto permitiría, a mi entender, a la vez reducir la brecha digital, entendida ésta como desigualdad en el uso de las potencialidades ofrecidas por las máquinas y en el acceso a la información (según las personas, las comunidades o los países) y mejorar la eficiencia de la comunicación mediada por ordenador o cualquier otro dispositivo electrónico en lo que respecta a la construcción de la identidad y de las relaciones sociales en el ciberespacio.

En este sentido, convendría también desarrollar un metalenguaje digital que permita reflexionar sobre las normas y usos digitales, al margen del que usamos para explicar las lenguas naturales fuera del ciberespacio. $\mathrm{O}$, mejor dicho, convendría continuar desarrollándolo, pues podemos considerar que el Análisis del Discurso digital, tal como lo vienen haciendo autores como, por ejemplo, Rodney Jones (2015), y Marie-Anne Paveau (2017), constituyen ya, en cierto modo, una forma de metalenguaje digital.

\section{BIBLIOGRAFÍA}

AREA MOREIRA, Manuel. “Una breve historia de las políticas de incorporación de las tecnologías digitales al sistema escolar en España". Quaderns digitals: Revista de Nuevas Tecnologías y Sociedad, n. 51, 2008, s/p [Disponível em: https://dialnet.unirioja. es/servlet/articulo?codigo=2566567. Accesso em: abr. 2019]

ASSOULINE, Pierre. "La métamorphose du lecteur", Le Débat, n. 170, p. $78-89,2012 / 3$.

BAKHTINE, Mikhail. Marxisme et philosophie du langage. Paris: Minuit, 1977 (1 ${ }^{\mathrm{a} e d}$. Leningrad 1929).

BALANDIER, Georges. Le Détour: Pouvoir et modernité. Paris: Fayard, 1985. 
BARON, Georges-Louis; BRUILLARD, Éric. “Technologies de l'information et de la communication et indigènes numériques: quelle situation?". Sciences et Technologies

de l'Information et de la Communication pour l'Éducation et la Formation, n. 15, s/p, 2008. Disponível em: http://sticef.org. Acesso em: abr. 2019.

BERNARDOT, Marc. "Plongée dans les métaphores et représentations liquides de la société numérique". Netcom, n. 32, v. 1-2, p. 29-60, 2018. Disponível em: https://journals. openedition.org/netcom/2886. Acesso: abr. 2019.

BÉTRANCOURT, Mireille. "L'ergonomie des TICE: quelles recherches pour quels usages sur le terrain ?" In: CHARLIER, B.; PERAYA, D. (Org.), Regards croisés sur la recherche en technologie de l'éducation. Bruxelles: De Boeck, 2007, p. 77-89.

BOURDELOIE, Hélène. "L'appropriation des dispositifs d'écriture numérique: translittératie et capitaux culturel et social". Études de communication, n. 38, p. 23-36, 2012.

BOYER, Priscilla; LEBRUN, Monique; ROY, Normand. "La grammaire et le numérique: des premiers pas hésitants". Revue de Linguistique et de Didactique des Langues, n. 56, s/p, 2017. Disponível em: <http://lidil.revues.org/4747>. Acesso em: abr. 2019.

BROTCORNE, Périne et al. Diversité et vulnérabilité dans les usages des TIC: La fracture numérique au second degré. Gent: Academia Press, 2010.

BUCKINGHAM, David. Media Education: Literacy, Learning and Contemporary Culture. Cambridge: Polity, 2003.

“Cultura Digital, Educação Midiática e o Lugar da Escolarização". Educação \& Realidade, n. 35 v. 3, p. 37-58, 2010.

CAELEN, Jean. "Interaction et multimodalité", In: BRUILLARD, E.; BALDNER, J.-M.; BARON, G.-L. Hypermédias et Apprentissages, Châtenay-Malabry: EPI-INRP, 1996, p. 11-32. 
DOUEIHI, Milad. La grande conversion numérique. Paris: Seuil, 2008. Pour un humanisme numérique, Paris: Seuil, 2011.

DUGUET, Amélie. Les pratiques pédagogiques en première année universitaire: description et analyse de leurs implications sur la scolarité des étudiants. Presses de l'Université de Bourgogne, 2014. Disponível em: https://tel.archives-ouvertes.fr/tel-01217315/ document. Acesso em: abr. 2019.

FALGAS Julien. "Needle, une innovation issue des SIC face à la crise de l'inspiration". Actes du XXIe Congrès de la SFSIC. Création, créativité et médiations. v. 3, Paris: SFSIC,2018, p. 221236. Disponível em: http://www.sfsic.org. Acesso em: abr. 2019.

FOUCHER, Anne-Laure; YUNN, Hyeon. "Être indigène numérique, utilisateur de Facebook et futur enseignant de FLE". Canadian Journal of Learning and Technology, n. 42, v. 4, s/p, 2016. Disponível em: https://www.cjlt.ca/. Acesso em: abr. 2019.

GUICHON, Nicolas. "Les usages des TIC par les lycéens déconnexion entre usages personnels et usages scolaires". Sciences et Technologies de l'Information et de la Communication pour l'Éducation et la Formation, n. 19, s/p, 2012. Disponível em: http://sticef.org. Acesso em: abr. 2019.

HARVEY Pierre-Léonard. Cyberespace et communautique: appropriation, réseaux, groupes virtuels. Sainte-Foy: Presses de l’Université Laval, 1995.

JONES, Rodney H. "Discourse, cybernetics, and the entextualization of the self". In: JONES, R. H.; CHIK, A.; HAFNER, C. (orgs.) Discourse and Digital Practices: Doing discourse analysis in the digital age. Routledge, London, 2015, p. 28-47.

KLEIN Annabelle. "Facebook quand tu nous tiens". In: PROULX, S.; MILLETTE, M.; HEATON, L. Médias sociaux. Enjeux pour la communication. Québec: Presses universitaires du Québec, 2012, p. 105-116. 
LATZKO-TOTH, Guillaume; PROULX, Serge. "Appropriation des technologies". In: PRUD'HOMME, Julien; DORAY, Pierre; BOUCHARD, Frédéric. (orgs.) Sciences, technologies et sociétés de A à Z. Montréal: Presses de l'Université de Montréal, 2015, p. 24-26.

LEVY Pierre. L'intelligence collective: pour une anthropologie du cyberespace, Paris: la Découverte, 1997.

LICOPPE, Christian (org.). L'évolution des cultures numériques: De la mutation du lien social à l'organisation du travail. Paris: FYP Éd., 2009.

MARCOTTE Jean-François. “Communautés virtuelles et sociabilité en réseaux: pour une redéfinition du lien social dans les environnements virtuels". Esprit critique, n. 5 v. 4, s/p, 2003. Disponível em: http://www.espritcritique.fr. Acesso em: abr. 2019.

MARCUSCHI, Luiz Antônio. Da fala para a escrita: atividades de retextualização. São Paulo: Cortez, 2001a.

" "O hipertexto como um novo espaço de escrita em sala de aula". Linguagem E Ensino, n. 4, v. 1, pp. 79-111, 2001b

MOATTI Michel. "Le numérique rattrapé par le digital?", Le Débat, n. 188, v. 1, s/p, 2016. Disponível em: http://data.overblog-kiwi.com/. Acesso em: abr. 2019.

- La Vie cachée d'Internet: Réseaux, tribus, accros. Paris: Imago, 2002.

MUSSO, Pierre. "La révolution numérique: techniques et mythologies". La Pensée, n. 355, pp.103-120, 2008.

PATEL, Namrata et al. “Une approche hybride pour la détection d'influenceurs dans les médias sociaux". In: ROUSSEY, Catherine (org.). Actes des 28es Journées francophones d'Ingénierie des Connaissances. Caen: IC, 2017, p. 115-120. Disponível em: https://pdfs.semanticscholar.org/. Acesso em: abr. 2019.

PAVEAU, Marie Anne. "Les textes numériques sont-ils des textes?". Itinéraires (Textualités numériques), n. 2014, v. 1, s/p, 2015. Disponível em: http://journals.openedition.org/ itineraires/2312. Acesso em: abr. 2019. 
L'analyse du discours numérique. Dictionnaire des formes et des pratiques, Paris, Editions Hermann, 2017.

PERAYA, Daniel; BONFILS, Philippe. “Détournements d'usages et nouvelles pratiques numériques: l'expérience des étudiants d'Ingémédia à l'Université de Toulon". Sciences et Technologies de l'Information et de la Communication pour l'Éducation et la Formation, n. 21, p. 239-268, 2014.

PLANTARD, Pascal. Anthropologie des usages du numérique. Tesis doctoral (Université de Nantes) Nantes: Université de Nantes, 2014. Disponível em: https://halshs.archives-ouvertes. fr/. Acesso em: abr. 2019.

PRENSKY, Marc. “Digital Natives, Digital Immigrants". On the Horizon, n. 9, v. 5, p. 1-6, 2001.

RHÉAUME, Jacques. "Les hypertextes et les hypermédias". In: KASZAP, M.; JEFFREY, D.; LEMIRE, G. (orgs.) Exploration d'internet, recherches en éducation et rôle des professionnels de l'enseignement. Paris: L’Harmattan, 2001, p. 189-206.

RHEINGOLD Howard. Les communautés virtuelles. Paris: Addison-Wesley, 1992.

SCARDIGLI, Victor. Les sens de la technique. Paris: PUF, 1992.

SIEMENS, George; GAŠEVIĆ, Dragan; DAWSON, Shane. Preparing for the Digital University: a review of the history and current state of distance, blended, and online learning. Arlington: Link Research Lab. 2015, s/p Disponível em: http:// linkresearchlab.org/PreparingDigitalUniversity.pdf. Acesso em: abr. 2019.

STIEGLER, Bernard (org.). Digital Studies: organologie des savoirs et technologies de la connaissance. Limoges: Fyp éditions, 2014.

SZUNDY, Paula; CARRÈRA, Tatianne; NASCIMENTO, Luciana Marino. "Leitores-navegantes de textos e hipertextos da literatura". Gragoatá, n. 40, v. 1, p. 354-379, 2016. 
TRICOT, André et al. "Utilité, utilisabilité, acceptabilité : interpréter les relations entre trois dimensions des EIAH". In: DESMOULINS, C.; MARQUET, P.; BOUHINEAU, D. (Orgs.), Environnements informatiques pour l'apprentissages humain. Paris: ATIEF-INRP, 2003, p. 391-402.

WALLON, Emmanuel. "La démocratisation culturelle, un horizon d'action". Les Cahiers français, n. 348, p. 79-86, 2009.

WOODS David; SIDNEY, Dekker. "Anticipating the effects of technological change: A new era of dynamics for human factors". Theoretical Issues in Ergonomics Science, n. 1, v. 3, p. 272-282, 2000.

\section{Resumo}

Identidade e língua no ciberespaço: existe uma consciência linguística digital?

Na ordem do ciberespaço, muitos internautas se autodenominam nativos ou indígenas digitais, constituindo uma nova comunidade étnica fora das fronteiras territoriais e culturais do mundo físico. Eles têm em comum o que poderíamos chamar de a mesma "consciência digital". Neste artigo, estudamos como essa consciência digital se manifesta por meio das expressões que os usuários do Twitter empregam para falar sobre quem são e o que fazem quando se comunicam por computador ou outros dispositivos conectados à rede da Internet. Nosso objetivo é tentar sistematizar algumas características específicas da linguagem digital, a fim de propor algumas pistas para sua pedagogia e participar do debate do "bom uso" da linguagem digital no contexto da cortesia digital.

Palavras-chave: língua digital linguística; consciência digital; cortesia digital; Twitter. 
Identidad y lengua en el ciberespacio: ¿existe una conciencia lingüística digital?

\section{Abstract \\ Identity and language in cyberspace: is there a digital linguistic conscience?}

In the cyberspace order, many netizens call themselves digital natives, constituting a new ethnic community outside the territorial and cultural boundaries of the physical world. They have in common what we might call the same "digital awareness". In this article we study how this digital awareness manifests itself through the expressions that Twitter users use to talk about who they are and what they do when they communicate by computer or other devices connected to the Internet network. Our goal is to try to systematize some specific characteristics of the digital language, in order to propose some clues for its pedagogy and to participate in the "good use" debate of the digital language within the framework of digital courtesy.

Keywords: Digital Language; Digital Awareness; Digital Courtesy, Twitter. 